\title{
Empresa social \\ basada en alianzas. \\ El caso del mercado de saneamiento como modelo de cambio e innovación social
}

\author{
ALEJANDRO FLORES CASTRO, Ph.D. ${ }^{1,2}$ \\ Profesor principal, Universidad del Pacífico, Perú. \\ flores_ja@up.edu.pe
}

\section{RESUMEN}

Las oportunidades para mejorar la calidad de vida en la base de la pirámide pueden ser capitalizadas, además de la subvención del Estado, por las alianzas de tipo Público-Privado-Social (PPs). El objetivo de este trabajo es diseñar un nuevo modelo de empresa -la empresa social- que cubre lo que el Estado y el sector privado solos no logran alcanzar. La propuesta está basada en alianzas con diferentes stakeholders, y se plantea un modelo de empresa para promover el acceso a servicios de saneamiento sostenible de calidad, a partir de incluir el cambio como elemento sustancial con el fin de lograr un paradigma nuevo en la concepción del acceso a servicios de saneamiento en la base de la pirámide.

Palabras clave. Empresa social; calidad de vida; alianzas; cambio; generación de valor.

Recibido: 30-jun-11, corregido: 27-abr-12 y aceptado: 26-jun-12

Clasificación JEL: M14; M19

$\infty$

\footnotetext{
1 Este documento fue seleccionado en la convocatoria para enviar artículos, Call for Papers, realizada en el marco del “II Simposio Iberoamericano de Estudios Gerenciales: Una mirada interdisciplinar a la innovación”, organizado por la revista académica Estudios Gerenciales bajo la dirección de la Facultad de Ciencias Administrativas y Económicas de la Universidad Icesi; el evento tuvo lugar los días 12, 13 y 14 de octubre de 2011, en la ciudad de Cali (Colombia). Este documento fue presentado en las sesiones simultáneas del área de "Innovación en relaciones organizacionales". 2 Dirigir correspondencia a: Av. Salaverry 2020, Jesús María, Lima, Perú.
} 
Social enterprise based on alliances. The case of the sanitation market as a model of social change and innovation

The opportunities to improve the quality of life at the base of the social pyramid can be tapped thanks not only to government grants, but also to public-private-social (PPS) alliances. The objective of this work is to design a new business model - a social enterprise - which meets the needs that the state and the private sector cannot provide on their own. The proposal is based on alliances with various stakeholders, suggesting a business model that promotes access to sustainable quality sanitation services by incorporating change as a substantial component in order to achieve a new paradigm with regards to the conception of access to sanitation services to those at the base of the social pyramid.

Keywords. Social enterprise; quality of life; partnerships; change; value generation.

\section{en}

\section{Empresa social baseada em parcerias. O caso do mercado de saneamento como modelo de mudança e de inovação social}

As oportunidades para melhorar a qualidade de vida na base da pirâmide podem ser capitalizadas, para além da subvenção do estado, pelas parcerias de tipo público-privado-social (PPS). O objetivo deste trabalho é conceber um novo modelo de empresa - a empresa social-que abranja o que o Estado e o setor privado sozinhos não conseguem alcançar. A proposta é baseada em parcerias com diferentes partes interessadas, e é proposto um modelo de empresa para promover o acesso a serviços de saneamento sustentável de qualidade, a partir da inclusão da mudança como um elemento substancial, a fim de alcançar um novo paradigma na concepção do acesso aos serviços de saneamento na base da pirâmide.

Palavras-chave. Empresa social; qualidade de vida; alianças; mudança; geração de valor. 


\section{Introducción}

A pesar de vivir en un contexto globalizado, no todas las personas tienen la oportunidad de construir su propio futuro para una mejor calidad de vida. Por ello, los organismos internacionales de apoyo al desarrollo juegan un rol vital en vista de que crean las condiciones para que los gobiernos nacionales, las instituciones públicas, las empresas privadas y el sector social, sienten las bases para el cambio en las poblaciones con menores recursos.

Desde los planteamientos de la Carta de Ottawa para la promoción de la salud (World Health Organization -wHo, 1986), hasta la Carta de Bangkok (wHO, 2005), la necesidad de brindar a la población menos favorecida una mejor calidad de vida, ha sido promovida por la Organización Mundial de la Salud con la finalidad de que los individuos puedan alcanzar una mejor posición en el contexto de los sistemas de cultivo y valores en que viven, y en relación con sus objetivos, expectativas, estándares y preocupaciones (Sirgy, Michalos, Ferriss, Easterling, Patrick \& Pavot, 2006).

Es así que uno de los aspectos de mejora, según los Objetivos del Milenio, corresponde a la cobertura de saneamiento que deben tener las personas en su vivienda, ya que aproximadamente unos 2.600 millones de personas en el mundo (unos 6,5 millones en el Perú) no cuentan con los medios suficientes y necesarios para vivir en una cultura de saneamiento. Tristemente, para estas personas, su única alternativa como estilo de vida es el defecar al aire libre.

Es por ello que alcanzar los Objetivos del Milenio, en el caso de la cobertura de saneamiento, implica necesariamente un proceso de cambio, demanda pensar y actuar de una forma diferente, requiere un paradigma nuevo que privilegie principios fundamentales del ser humano como la dignidad y el derecho a la intimidad de las personas para llevar a cabo actos tan humanos como eliminar sus excretas sin el peligro de contaminar. Pero también demanda la participación de múltiples actores en la generación de las oportunidades para que las personas tengan mejores condiciones para el acceso al saneamiento en sus viviendas. Se requiere que los sectores público, privado y social logren sinergias, trabajando mediante una alianza social con el objeto de presentar a la sociedad una oferta integral de saneamiento que facilite el acceso a las diferentes oportunidades y alternativas de saneamiento existentes.

Por el lado de la demanda, se requiere estimular y promover una cultura de saneamiento con una estrategia de comunicación que enseñe a las personas los beneficios de vivir en condiciones de salubridad. El alineamiento de la oferta y demanda en estas condiciones, favorecerán el desarrollo de un mercado de saneamiento para pobladores de bajos ingresos que genere, a su vez, las oportunidades para crear mejores condiciones de vida.

En este sentido, a la luz de los alcances de las experiencias piloto de la iniciativa Creando Mercados de Saneamiento ${ }^{3}$ (CMs) en el Perú, entre el 2007 y el 2010, se revisan en este documento aquellos factores determinantes que dan sustento a un modelo de empresa social basado en alianzas. Se trata pues de un modelo que integra a múltiples agentes sociales y que, a su vez, busca incrementar el acceso a los servicios de saneamiento en la vivienda a partir de un nuevo paradigma, el de la creación de un

3 Iniciativa integrada por el gobierno peruano, Sedapal, el programa de Agua y Saneamiento del Banco Mundial, la Agencia Suiza para el Desarrollo y la Cooperación, el AID y la Fundación Ensamble. 
mercado de saneamiento en la población de bajos ingresos.

A continuación se realiza una revisión de la literatura acerca de la empresa social en el marco del rol del empresario, para luego presentar la iniciativa Creando Mercados de Saneamiento y dar a conocer el nivel de accesibilidad del saneamiento en el Perú. Finalmente se presentan las principales conclusiones y recomendaciones del estudio.

\section{La empresa social en contexto}

Para hablar de empresa social es necesario mencionar al empresario puesto que es el sujeto que visiona, diseña y define la naturaleza y la razón de ser del instrumento o del medio, es decir, de la empresa en sí. El rol del empresario ha sido siempre el mismo: capitalizar oportunidades, innovar y generar valor. Una revisión de la literatura desde Cantillón (1755), pasando por Schumpeter (1934), Coase (1937), Drucker (1985), Stevenson \& Gumpert (1985), Hébert \& Link (1989, 2006), Stevenson (2006) y van Praag \& Versloot (2007), confirman esta afirmación.

Si esto es así, ¿deja de tener sentido o cambia el rol del empresario? La respuesta es no, pues el rol sigue siendo el mismo, así como el sentido de su participación en la economía de la sociedad. Entonces ¿qué cambia en el enfoque de una empresa social? Cambian la visión, la naturaleza y la razón de ser del instrumento que el empresario crea; cambian, asimismo, los fines por los cuales la empresa es creada. De esta manera, la empresa es idealizada, visionada y creada con una finalidad que la hace diferente de una con fines de lucro o un negocio, con una finalidad de capitalizar oportunidades para crear un cambio en la sociedad (Mair \& Marti, 2004; Wei-Skillern, Austin, Leonard \& Stevenson, 2007). Bajo esta perspectiva, se plantea el modelo de empresa social basada en alianzas, donde la generación de valor está determinada por el beneficio colectivo que se alcanza cuando se integra a diferentes stakeholders en un objetivo común, es decir, el que plantea la empresa social (Weerawardena \& Mort, 2006).

La perspectiva de plantear una empresa social basada en alianzas descansa en el ahorro de costes de transacción (Coase, 1937) que se generan en un mercado incipiente o inexistente, como en el caso del mercado de saneamiento en la base de la pirámide. Las escasas transacciones en este tipo de mercado no representan suficiente incentivo económico para una empresa con fines de lucro, por lo que un modelo de empresa social (basada en relaciones atemporales para concertar oferta y demanda, para generar economías de escala e incentivar la formación de un mercado de mano de obra que asuma la instalación de los bienes; es decir, basada en alianzas) se yergue como una opción.

\section{Acerca de la iniciativa Creando Mercados de Saneamiento (cMs)}

Alineado con las propuestas de la promoción de la salud de la Carta de Ottawa (who, 1986), en lo referente a la reorientación de los servicios sanitarios y a la convocatoria a la participación comunitaria, así como a los planteamientos de la Carta de Bangkok (wHO, 2005), encaminados a un mayor compromiso para mejorar la salud de los individuos buscando cerrar las brechas aún existentes a través de políticas públicas alianzas para la acción; el gobierno peruano, para el período 2006-2015, se propuso incrementar la cobertura de los servicios básicos de saneamiento del $59 \%$ al $77 \%$. En este sentido, la iniciativa Creando Mercados de Saneamiento (CMs) busca estimular el desarrollo del mercado del saneamiento para la población de bajos ingresos en cuatro 
zonas piloto en el país (World Bank, 2010), ${ }^{4}$ considerando los factores que estimulan la inversión en saneamiento en la base de la pirámide presentados en el Anexo 1.

Esta iniciativa tiene por finalidad el promover las buenas prácticas de higiene y de servicios de saneamiento, así como motivar a la población en el uso de servicios de higiene apropiados, a través de la integración de la oferta y la demanda del mercado de saneamiento destinado a sectores de población de bajos ingresos. Dicha integración se realiza desde una perspectiva de la participación de la comunidad local, de los usuarios finales de los servicios de saneamiento, de los proveedores de servicios y de bienes y equipos de sistemas de saneamiento domiciliario, así como de los líderes comunales y autoridades locales interesados en el bienestar y saneamiento de su comunidad.

Los resultados del programa piloto de la iniciativa CMs permiten delinear un nuevo paradigma para mejorar la calidad de vida de pobladores de bajos ingresos en la perspectiva de una alianza social con la participación del Estado, de los organismos internacionales de apoyo, de los gobiernos regionales y locales, así como con la intervención de líderes comunales y de la empresa privada.

4 Realizada en las localidades de Callao, Cajamarca, Cuzco y Ancash.

\section{Accesibilidad al saneamiento en el Perú}

Perú, con una población de 29.797.694 personas y un total de 6.400 .000 viviendas, registraba en el 2007 un total de 2.621.255 viviendas $(41 \%)$ con carencias de servicio higiénico conectadas a la red pública de desagüe a nivel nacional (Instituto Nacional de Estadística e Informática del Perú -INEI, 2007) (ver Tabla 1). Esto representa, aproximadamente, unas 6,5 millones personas que no tienen acceso a servicios de saneamiento donde un $43 \%$ de ellos viven en las zonas urbanas y el $57 \%$ en las rurales.

Si bien ha existido un crecimiento en la atención de las necesidades de saneamiento, entre los años 1993 y 2007, lo cierto es que de acuerdo a los resultados del censo del 2007, aproximadamente un tercio de la población en el Perú no tenía acceso al saneamiento. Es así que, del $81 \%$ de personas que acceden a servicios básicos de saneamiento, tan solo el $22 \%$ lo hace a través de letrinas de pozo ciego. Ello no representa -en ningún caso- una mejora en la calidad de vida de las personas, puesto que las condiciones de las letrinas son precarias y no garantizan ni la privacidad ni la seguridad o el confort para los usuarios.

\section{1 Ámbito de intervención}

El programa piloto se desarrolló en las localidades de Cajamarca, Ancash, Cuzco y Callao. En la Tabla 2 se detallan las zonas

Tabla 1. Número de viviendas en el Perú sin acceso a servicios de saneamiento

\begin{tabular}{lcc}
\hline \multirow{2}{*}{ Viviendas sin conexión a red pública de desagüe } & \multicolumn{2}{c}{ Ámbito } \\
\cline { 2 - 3 } & Urbano & Rural \\
\hline Acequia, sin acceso & $499.154,00$ & $725.699,00$ \\
Letrinas / pozo ciego & $634.579,00$ & $761.823,00$ \\
Viviendas sin conexión a red pública de desagüe & $1.133 .733,00$ & $1.487 .522,00$ \\
\hline Total viviendas a nivel nacional sin conexión & \multicolumn{2}{c}{$2.621 .255,00$} \\
\hline
\end{tabular}

Fuente: Elaboración propia con base en INEI (2007). 
Tabla 2. Ámbitos de intervención y resultados de ventas

\begin{tabular}{lccc}
\hline Zona piloto & $\begin{array}{c}\text { Comunidades } \\
\text { intervenidas }\end{array}$ & Población & Baños vendidos \\
\hline Cajamarca & 5 & 5,985 & 79 \\
Ancash & 5 & 1,934 & 65 \\
Cuzco & 15 & 6,758 & 145 \\
Callao & 8 & 130,000 & 67 \\
\hline
\end{tabular}

Fuente: Adaptado de World Bank (2010).

de intervención y los resultados de ventas de servicios de saneamiento (baños), logradas en la fase piloto. La generación y desarrollo de mercados inclusivos no se obtiene de manera espontánea, por lo que se requiere la intervención de un agente catalizador de procesos que facilite el impulso inicial y una progresiva dinamización. Para tal fin, se trabajó con diferentes organizaciones no gubernamentales, las que llevaron a cabo los procesos de intervención directa con la comunidad.

\subsection{Objetivos y componentes}

Las iniciativas fueron implementadas por las organizaciones Adra Perú y Care Perú. Tuvieron como objetivo incrementar en los distritos y comunidades seleccionadas, el acceso a un sistema sanitario seguro, sostenible y de bajo costo (ver Tabla 3 ).

\section{El mercado de saneamiento en la base de la pirámide}

\subsection{Perfil del consumidor-usuario}

Los consumidores-usuarios en la base de la pirámide son todas aquellas personas que no gozan de los servicios de saneamiento en sus domicilios y que ven restringidas sus libertades de niveles de vida de acuerdo a los planteamientos de Sen (1984), dada las limitadas capacidades para evitar ver vejada su dignidad humana, de tal manera que ponen en riesgo su salud y la de todos los miembros de su familia. Este colectivo puede ser estratificado de acuerdo a sus aspiraciones de mejora de la calidad de vida, ya sea porque tienen como referentes a familiares cercanos o porque tuvieron la oportunidad de conocer una realidad distinta a la que viven.

\subsection{Diseño del producto/servicio}

En el caso del mercado de saneamiento, el concepto de producto no solo está definido por el bien físico denominado baño o por sistemas y equipos de saneamiento con agua y sin agua de uso domiciliario. Además, se deben incluir otros aspectos como la habilitación del lugar, la instalación del bien y el financiamiento, que se deben contemplar para que el usuario finalmente pueda disfrutar del baño.

\subsection{Los canales de comercialización}

Dada las características del producto, así como las del mercado que se desea desarrollar, es necesario estructurar un canal de distribución de los paquetes de saneamiento en las zonas urbanas y rurales, con el fin de facilitar el acceso a los usuarios. Se requiere un mecanismo de abastecimiento y almacenaje de los equipos de saneamiento dado que, al no existir un mercado totalmente desarrollado, la frecuencia de compra en un inicio es muy baja. Se necesita articular una red de pequeñas ferreterías o negocios 
Tabla 3. Objetivos, estrategia y acciones de la iniciativa CMS

\begin{tabular}{ll}
\hline \multicolumn{1}{c}{ Elementos del modelo } & \multicolumn{1}{c}{ Contenidos } \\
\hline Objetivo & $\begin{array}{l}\text { Incrementar el acceso de la población a facilidades sanitarias seguras, } \\
\text { sostenibles y de bajo costo que mejoren sus condiciones de salud y dis- } \\
\text { minuyan el impacto ambiental negativo. }\end{array}$ \\
Acciones específicas & $\begin{array}{l}\text { Promoción de mercados de saneamiento locales } \\
\text { Movilización comunitaria. }\end{array}$ \\
& Movilización educativa. \\
& Generar demanda efectiva continua en el espacio intra-domiciliario. \\
& $\begin{array}{l}\text { Desarrollar una oferta diversificada de opciones tecnológicas económica- } \\
\text { mente accesibles. }\end{array}$ \\
& $\begin{array}{l}\text { Generar opciones económicas y financieras formales o autogeneradas } \\
\text { para el financiamiento de saneamiento de calidad. }\end{array}$ \\
& $\begin{array}{l}\text { Promover la concertación y alianza de tipo público, privado y social. } \\
\text { Venta de sistemas de saneamiento para domicilio. }\end{array}$ \\
Resultados esperados & Instalación de sistemas de saneamiento para domicilio. \\
\hline
\end{tabular}

Fuente: Adaptado de World Bank (2010).

de insumos para la construcción que lleguen a compartir los riesgos y costos de una iniciativa empresarial de este tipo. Al respecto, es importante que se concentre la oferta de los insumos y equipos para que los mismos estén al alcance de los usuarios.

\subsection{Variable del precio}

De acuerdo a los datos proporcionados por el sistema de monitoreo del proyecto CMs, correspondiente al mes de mayo de 2009, se estiman los valores monetarios (en USD\$) a los que los usuarios estarían dispuestos a invertir con la finalidad de acceder a un sistema de saneamiento (seco o hidráulico). Todo ello se expresa en la Tabla 4.

\subsection{Alternativas de financiamiento}

La facilidad al acceso al saneamiento se ve limitada por los bajos niveles de ingreso de los pobladores en las zonas donde se llevaron a cabo los estudios piloto. Esto representa, no obstante, una oportunidad para el diseño de novedosos esquemas financieros basados en mecanismos de crédito comunal que, a su vez, permitirían a los usuarios financiar la adquisición de insumos y equipos de saneamiento, ante la dificultad de costos altos y limitación en la liquidez inmediata para pagar la implementación completa del servicio de saneamiento.

\subsection{La necesidad de una alianza Público- Privado-Social (PPS)}

Lograr un valor integrado en la base de la pirámide depende de la capacidad de una alianza PPs que permita articular las capacidades de los principales actores, quienes deben cumplir con sus roles para asegurar los objetivos de la misma, bajo el modelo de una alianza de integración (Austin, 2003). Solo la interacción dinámica que se genera 
Tabla 4. Precio de venta promedio por tipo de sistema (cifras en USD\$)

\begin{tabular}{lcc}
\hline Sistema de saneamiento con agua y sin agua a nivel domiciliario & Urbano & Rural \\
\hline Sistema seco (caseta con hoyo seco y losa) & 350 & 232 \\
Sistema hidráulico (letrina de arrastre hidráulico) & 650 & 690 \\
\hline
\end{tabular}

Fuente: Adaptado de World Bank (2010).

por la integración y la participación activa de los sectores públicos (gobiernos nacional, regional y local), los sectores privados (empresa privada y nuevos emprendimientos) y el sector social (colectivos familiares y las comunidades de base) - tal como se representa en el Gráfico 1-, aseguran la sostenibilidad de la alianza.

El objeto central de la alianza PPs es lograr que los hogares peruanos accedan a servicios básicos de saneamiento, en el marco de una vivienda digna, saludable y eco-eficiente. Dicha meta está especialmente dirigida a colectivos de bajos recursos comprendidos en la base de la pirámide.

La alianza PPs debe consolidar un modelo de negocio que genere valor para los usuarios basado en una serie de interacciones en el nivel local, que es el lugar donde realmente el mercado de saneamiento se desarrollará. No bastarán las coordinaciones en los niveles nacional o regional, si es que en el local no se sientan las bases para que el modelo interactúe.

En el ámbito local es donde los actores clave (promotor comunitario y operador financiero) tienen una mayor capacidad de generar la demanda en el mercado de saneamiento, por lo tanto, el eje central de la alianza y donde deberá conseguirse mayor sinergia social es en este nivel. En todo este contexto, queda claro que la articulación de la alianza PPS no se genera sola, sino que debe ser administrada y gestionada. Al respecto, en el Anexo 2 se presentan los logros de aprendizaje de la fase piloto de la iniciativa CMS.

\section{Generación de valor basada en el cambio}

Las oportunidades identificadas, tanto por las necesidades de desarrollo del mercado de saneamiento como por la presencia de determinados actores involucrados, a pesar de la nula existencia de una estructura formal de mercado, demandan una forma de intervención diferente. Esto sucede desde la perspectiva de un nuevo paradigma que busca introducir cambios en las relaciones con los diferentes actores, a fin de sustentar una nueva forma de generar valor.

El cambio en las organizaciones se da por la intervención directa e indirecta de determinadas variables, sobre las cuales los líderes pueden o no conocer su origen, su direccionamiento o tener control sobre ellas. Es así que toda manifestación de cambio se presenta como uno no esperado (sucede aleatoriamente), o como uno buscado (sucede intencionalmente). Desde una perspectiva unidimensional, el cambio puede ser representado de acuerdo al Gráfico 2.

Bajo esta misma perspectiva, pero con un mayor grado de complejidad, se puede realizar un análisis bidimensional del cambio, el cual comprende las cualidades de aleatoriedad y de intencionalidad. Estas cualidades corresponden a la forma como se genera el cambio según su nivel de influjo (sea mayor o menor) sobre el cambio. Cada alternativa posible, resultado de la matriz de doble entrada que se genera, se convierte en un escenario para la ocurrencia del cambio, en función de una mayor o menor intensi- 
Gráfico 1. Interacción dinámica de la alianza público-privado-social (PPs)

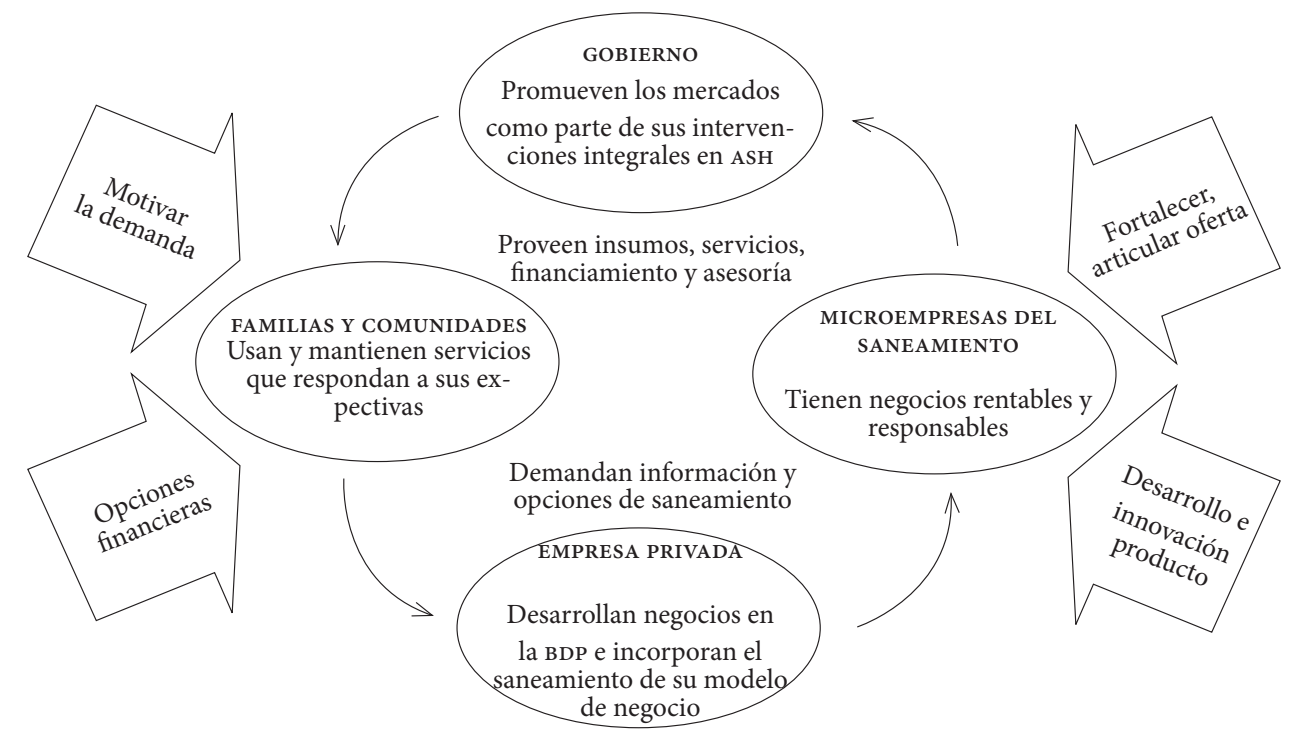

Fuente: Adaptado de World Bank (2010).

Gráfico 2. Escenario o ámbito de acción del cambio en las organizaciones

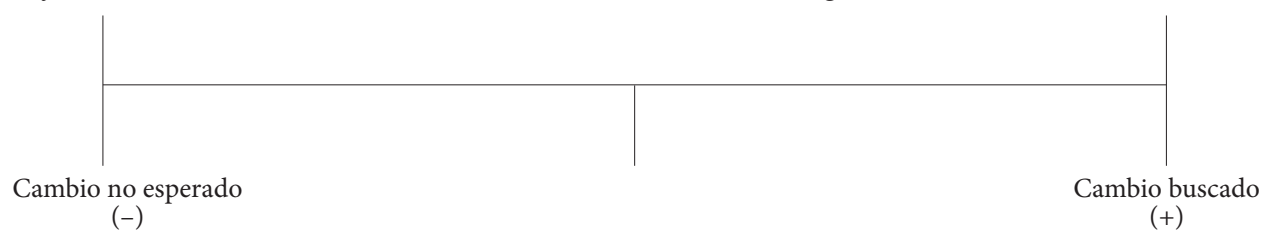

Fuente: Elaboración propia.

dad del influjo de cada variable, tal como se representa en el Gráfico 3.

\section{Matriz: condicionantes del cambio}

Bajo el contexto del enfoque bidimensional, se configura la matriz de condicionantes del cambio, donde este tendrá lugar en función del grado de aleatoriedad y del grado de intencionalidad. Esta matriz de doble entrada permite identificar las alternativas plausibles de ocurrencia del cambio que una organización puede implantar en un determinado momento. Así, se tiene el cambio predefinido, el deliberado, el conver- gente, $y$, desde luego, la opción de no iniciar ningún proceso de cambio. En el Gráfico 4 se presentan las alternativas plausibles de ocurrencia del cambio, donde las variables aleatoriedad e intencionalidad constituyen sus condicionantes.

En el Gráfico 4 se aprecia sobre el eje de abscisas, la dimensión de la aleatoriedad, es decir, una menor o mayor aleatoriedad en la ocurrencia del cambio que, en definitiva, significa un menor o mayor influjo sobre la organización y que le exige a esta una respuesta concreta. De igual manera, en el eje de ordenadas se representa la dimensión de 
Gráfico 3. Escenario o ámbito bidimensional de la acción del cambio

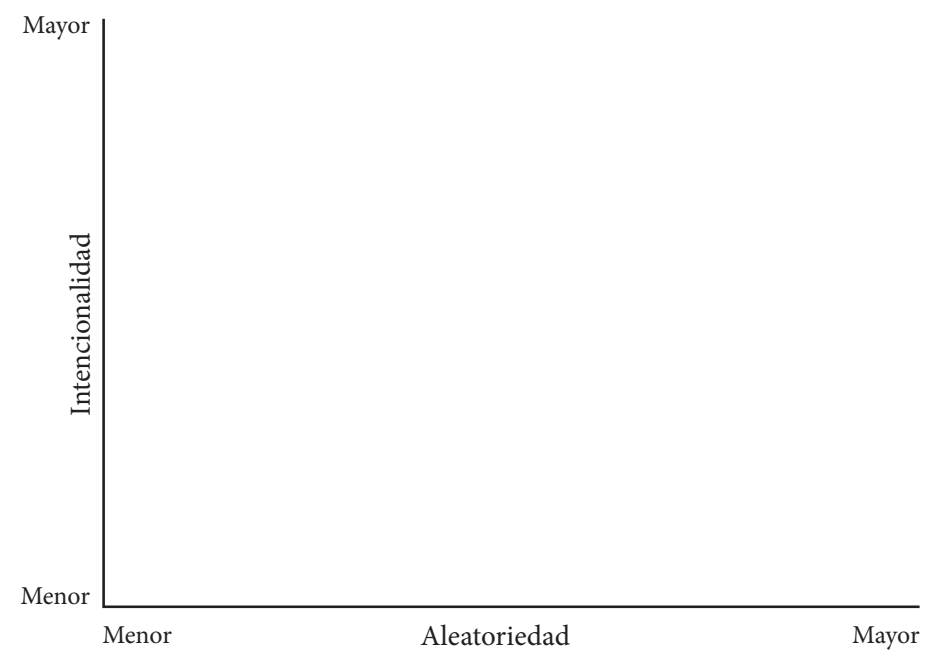

Fuente: Elaboración propia.

Gráfico 4. Matriz de condicionantes del cambio

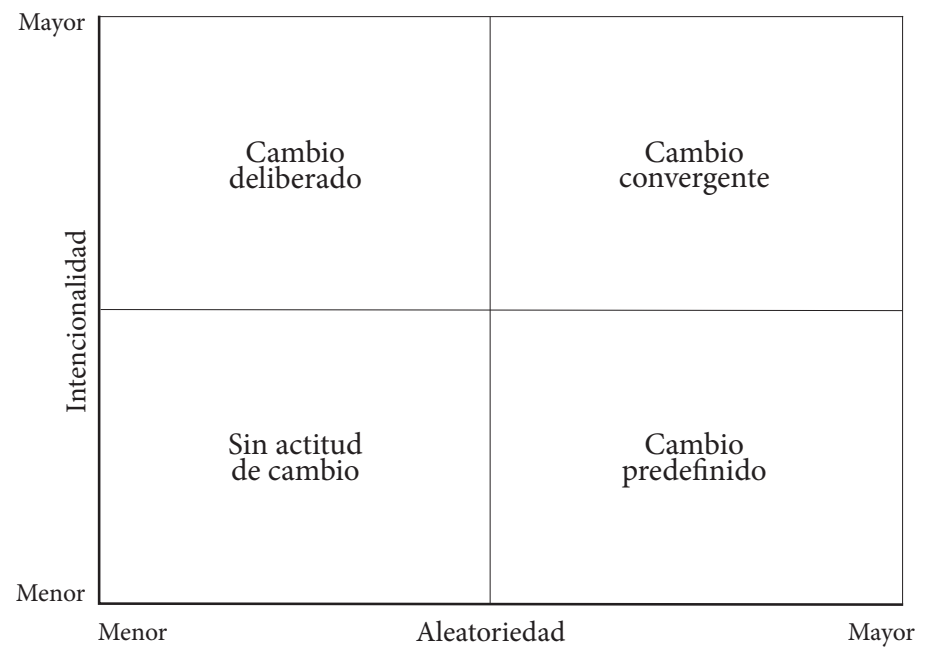

Fuente: Elaboración propia.

Sin embargo, es viable concebir un espacio donde las tres iniciativas anteriores puedan comulgar juntas para alcanzar un valor más vasto para la comunidad, un espacio donde se podrá pensar en una alternativa más inclusiva en la que estén presentes los diferentes actores. Estos, sin perder de vista sus expectativas, igualmente jugarán un rol determinante en la generación de valor en la sociedad que demanda el bienestar en el aspecto de saneamiento. Se trataría de un espacio donde se brinde la oportunidad de mejorar a las personas al desarrollar un mercado de saneamiento en la base de la 
la intencionalidad: una menor o mayor intencionalidad con que la organización busca alcanzar el cambio, lo que se traduce en un menor o mayor influjo de la organización, a partir de sus recursos y capacidades, sobre las condiciones del entorno. La incorporación del cambio en una empresa, organización o institución, sea este del tipo predefinido, deliberado o convergente, constituye una forma natural de actuar y responder de las organizaciones ante el influjo del entorno sectorial con la finalidad de adaptarse a él o definir un nuevo modelo de trabajo.

En el Anexo 3 se presentan las principales características de los tipos de cambio que una empresa puede implantar en su organización a partir de las condiciones bajo las cuales el cambio tiene oportunidad de ocurrir, en función de una mayor o menor intensidad del influjo de la variable intencionalidad o aleatoriedad.

\section{Generación de valor a partir de una alianza social}

En general, toda iniciativa de colaboración está destinada al éxito. Para que ello suceda, se deben atender dos instancias. Por una parte, los procesos de negociación entre las organizaciones involucradas, lo que permite alinear intereses particulares en busca de un beneficio singular compartido, principalmente económico, pero también de posicionamiento y de acceso a nuevos mercados.

Por otra parte, esto sucederá en la medida que la propuesta de valor generada por el esfuerzo de la colaboración satisfaga una necesidad latente o incipiente en el mercado (Celli \& González, 2010). En un contexto de colaboración entre organizaciones sin fines de lucro y empresas netamente comerciales, el sentido de misión común es más fuerte (Ogliastri, 2003), por lo que el grado de alineamiento entre las partes se vuelve un elemento esencial y determinante, aun cuan- do la propuesta de valor ofrecida sea de vital importancia para el mercado. En este sentido, que los socios de una alianza comprendan e interioricen la naturaleza de la colaboración se convierte en una tarea primordial y primigenia antes de iniciar cualquier esfuerzo de cooperación.

Por la naturaleza de la colaboración, las organizaciones pueden llegar a establecer alianzas de tipo filantrópica, transaccional y de integración (Austin, 2003), las mismas que pueden ser concebidas como un continuo de la colaboración, ${ }^{5}$ donde el alineamiento estratégico permitirá a las empresas identificar el beneficio singular que las lleve a colaborar.

Sin lugar a dudas, todas las iniciativas para alcanzar el bienestar en el aspecto de saneamiento tienen una clara orientación para generar valor en la sociedad; sin embargo, no todas siguen un mismo mecanismo o proceso. En este sentido, tan válida es la iniciativa privada que busca como principal beneficio la maximización de sus utilidades, como la iniciativa pública que busca mejorar el nivel de eficiencia de su gasto a partir de un factor incremental (tipo de cambio predefinido influenciado por una política pública). La primera podrá llevar a cabo su cometido mediante la dirección de sus acciones, fundamentalmente hacia el mercado, con la necesidad de altos desempeños a corto plazo, caracterizado, por lo general, en un tipo de cambio deliberado. También, se puede contemplar la iniciativa del sector social que persigue el bienestar y el progreso, pero cuyo accionar es más estructurado hacia el desarrollo a largo plazo (cambio deliberado).

5 El marco o Modelo Continuo de Colaboración de sarrollado por Austin (2003) puede ser utilizado como herramienta estratégica, sin embargo, esto no implica que cada alianza tenga que pasar por cada una de las tres etapas. 
pirámide (donde el tipo de cambio requerido se base, por su parte, en un cambio convergente).

Este esquema, basado en el modelo de Thakor \& Quinn (2006), permite plantear una propuesta de generación de valor a partir de una alianza social, en la que convergen los intereses de los sectores privado, público y social; $y$ en el efecto de sinergia que se logre desarrollar a largo plazo a través de la colaboración: "haciéndolo juntos" (Baskovich \& Ser'Jacobs, 2010), es decir, a través de una alianza público-privado-social (alianza PPs).

La propuesta de la alianza PPs desarrolla un efecto sinérgico superior a los otros plan- teamientos debido a que cuando se actúa solo, los resultados no llegan a maximizar la contribución de valor para todos los participes sociales, excepto para sus propios intereses: i) sector privado, con resultados a corto plazo para generar valor a partir de la competencia: "haciéndolo rápido"; ii) sector público, con desempeños incrementales para generar valor a partir del control: "haciéndolo bien"; y iii) el sector social, para generar valor a partir del progreso focalizado: "haciéndolo primero", tal como se presenta en el Gráfico 5.

La propuesta "haciéndolo juntos" corresponde a un modelo de cambio convergente y representa el logro de la sinergia social que

Gráfico 5. Modelo de generación de valor

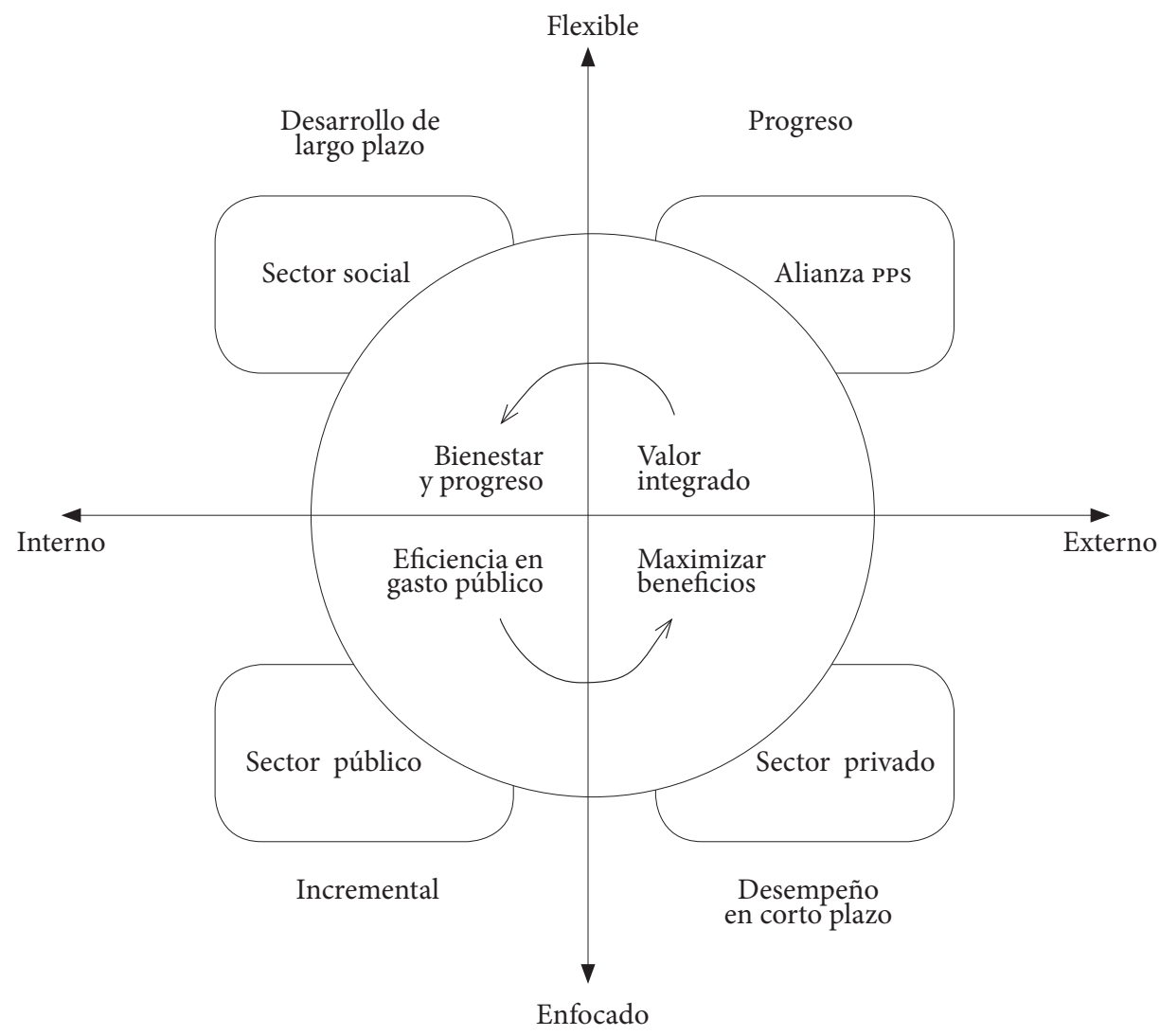

Fuente: De Creación de valor para el sector público, privado y social a través de la promoción de mercados de saneamiento en la base de la pirámide en el Perú (Tesis no publicada para optar al título de Magister en Administración, Universidad del Pacífico, Lima, Perú), por M.R. Baskovich \& A. Ser'Jacobs, 2010, p. 57. 
toda empresa debe alcanzar para asegurar su sostenibilidad y competitividad.

La alianza Pps tiene su sustento en el efecto sinérgico que se puede lograr alcanzando un valor integrado, el mismo que se basa en los recursos y capacidades de los actores públicos-privados-sociales, que en definitiva representan a los principales stakeholders de la alianza, que en el caso del mercado de saneamiento estarán representados por:

- Stakeholders públicos: integrados por el gobierno nacional, gobierno regional y gobierno local.

- Stakeholders privados: integrados por los proveedores de insumos de saneamiento y construcción, las microempresas del saneamiento, los artesanos o técnicos, los pequeños y micro negocios, los técnicos sanitarios, gasfiteros, maestros de obra, albañiles o ayudantes, las instituciones financieras reguladas y no reguladas, las empresas exportadoras, extractivas y de turismo.

- Stakeholders sociales: integrados por las familias y las comunidades, las organizaciones no gubernamentales y de voluntariado, los centros de investigación de universidades e institutos técnicos y la sociedad civil organizada.

\section{Cosmovisión de la alianza PPS}

Para alcanzar un valor integrado a partir de la concurrencia de las capacidades y recursos de los actores públicos-privadossociales, se requiere de una guía de acción que establezca qué es lo que se persigue, así como identificar los medios que se van a requerir para su implementación. Se trata de un nuevo modelo de negocio basado en múltiples alianzas, de una forma particular de entender las relaciones y las interacciones entre los diferentes actores, que por más que compartan un objetivo común, cada uno de ellos -de manera individual- desarrolla su propia visón de cómo alcanzarlo. Por ello, es preciso estructurar y plantear una nueva cosmovisión de cómo entender las relaciones entre sí, de manera que permita generar un nuevo paradigma, a través de la creación de un negocio social sustentado en la participación de los actores de una alianza PPs.

En el Gráfico 6 se presenta el conjunto de relaciones e interacciones básicas que deberá establecer la empresa social, cuya misión será el de promover el acceso a servicios de saneamiento sostenible de calidad en su zona de influencia.

\section{La presencia de multiactores}

La creación de un mercado de saneamiento requiere la participación de multi-actores que favorezcan la interacción de la oferta y demanda por los servicios de saneamiento para la vivienda. La dinámica de la interacción entre ofertantes y demandantes se genera y gestiona a través de la alianza PPs.

\section{La asociatividad como modelo de gestión}

Comprende aquellos mecanismos de cooperación entre varias instituciones (juntas vecinales, distintas ONG, empresas, instituciones públicas, etc.) donde cada participante, sin perder su independencia y autonomía gerencial, decide voluntariamente participar en un esfuerzo conjunto para alcanzar un objetivo común. Lo que pretende este modelo es la articulación de la oferta con la demanda para dar inicio a la dinámica natural de interacciones entre las partes. En este sentido, se propone un modelo de negocio que articule a los diferentes partícipes sociales, estimule la demanda y promueva una cultura de soluciones alternativas de saneamiento. El modelo de negocio que se propone contempla los siguientes aspectos clave para su concreción y sostenibilidad en el tiempo:

- Se trata de una iniciativa conjunta entre el sector privado, el gobierno local y la sociedad civil organizada. 
Gráfico 6. Red de relaciones en interacciones básicas de la empresa social

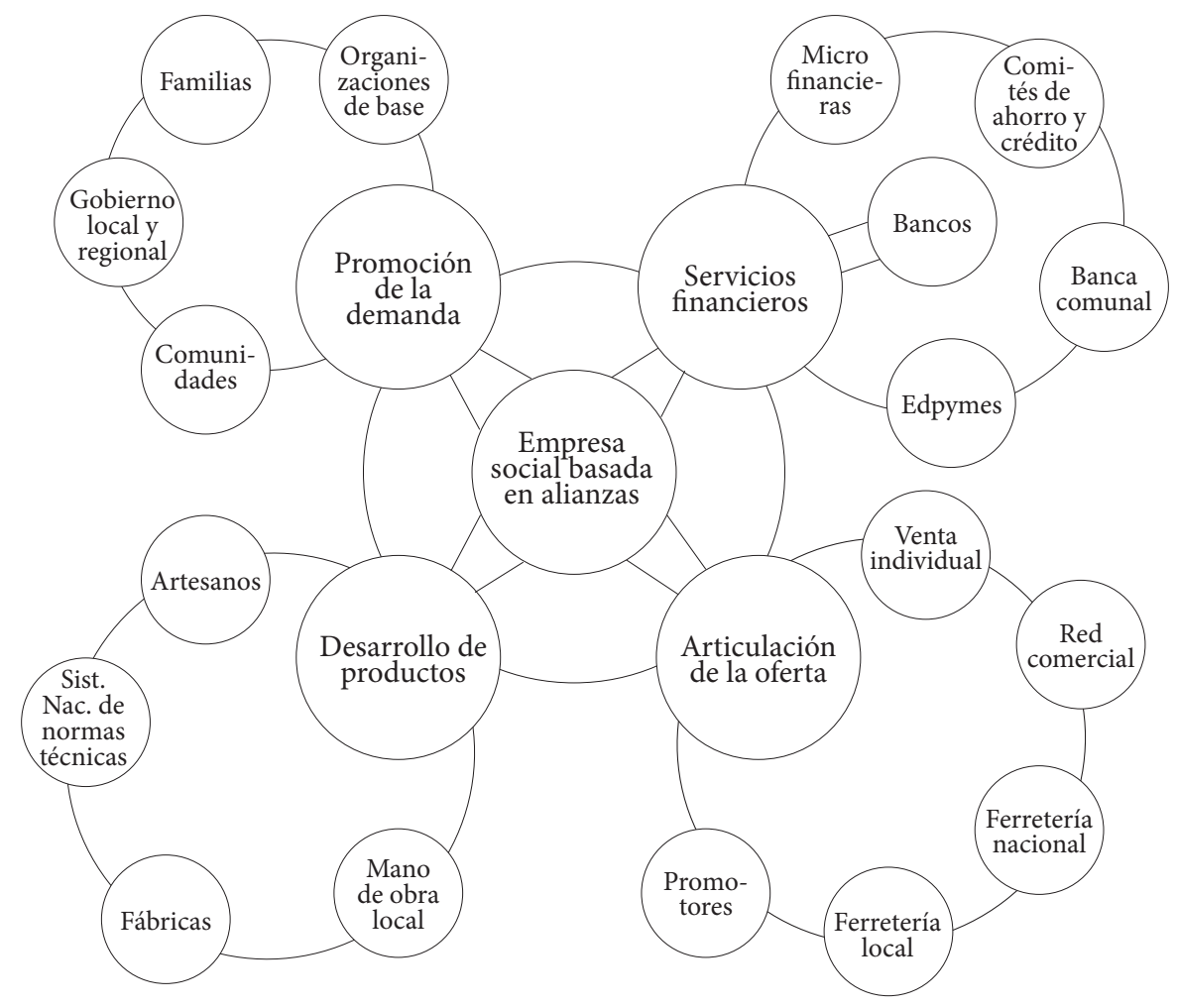

Fuente: Elaboración propia.

- Se enmarca en la implementación de las buenas prácticas de higiene y desarrollo de servicios de saneamiento, basado en el desarrollo de una cultura de saneamiento y de salud orientada a la población de bajos ingresos y en el acompañamiento y apoyo a la población para facilitarles el proceso de incorporación de una cultura de saneamiento y salud en su vida diaria.

- Se establecen alianzas que dinamicen una oferta integral de saneamiento a los consumidores locales, basadas en la integración del canal de distribución, de los proveedores de servicios y de la oferta de financiamiento.

\section{Conclusiones}

Los resultados de esta investigación permiten concluir que es posible alcanzar una mayor cobertura de saneamiento de las personas ubicadas en la base de la pirámide por medio de la implementación de empresas sociales basadas en alianzas pPs. El modelo de empresa social, basado en alianzas PPs, se diseña para generar sinergia social sustentada en la integración de los diferentes actores que participan en la iniciativa.

Así mismo, la empresa social está orientada a capitalizar oportunidades, innovar y generar valor en los mercados existentes de la base de la pirámide, como por ejemplo, el caso del mercado de saneamiento. Para lograr esto, es preciso introducir procesos de cambio de tipo convergente y, dadas las características de la alianza PPS, es preciso trabajar en el escenario "haciéndolo juntos", puesto que maximiza la generación de valor y favorecela asociatividad. 
Estos procesos de cambio, incorporando a diferentes stakeholders o participes sociales, exigen un mayor grado de comunicación y coordinación entre las partes lo que permite una mirada interdisciplinar y trabajar con un enfoque abierto de la innovación.

Los líderes de la comunidad y la sociedad civil organizada tienen, en el mecanismo de la asociatividad, un modelo de trabajo para poder avanzar en la mejora de la calidad de vida de sus comunidades.

Por su parte, poder gestionar el cambio se convierte en una de las principales limitaciones y restricciones para que los modelos de empresa social basada en alianzas lleguen a concretarse, especialmente por la necesidad de llegar a mantener un equilibrio entre los integrantes de la alianza. En este sentido, las empresas sociales solo aseguran su sostenibilidad en el tiempo en la medida en que todos los actores se sientan satisfechos con el logro de sus metas. Esta situación es altamente compleja si se analiza la conformación de las alianzas, donde la generación de valor en la componente pública se sustenta en factores totalmente diferentes a los de la componente privada, y no siempre alineados a los intereses sociales. Esto hace que su implementación y gestión se vuelva compleja y poco predecible.

Nuevas líneas de investigación deben encaminarse al logro de un mayor nivel de sostenibilidad de las empresas sociales, analizando las competencias y habilidades que debe generarse internamente entre los socios a fin de reducir la complejidad de su gestión y hacerlas más predecible.

\section{Referencias bibliográficas}

Austin, J.E. (2003). El desafío de la colaboración: cómo las organizaciones sin fines de lucro y las empresas comerciales alcanzan el éxito mediante alianzas estratégicas. Buenos Aires: Granica.

Baskovich, M.R. \& Ser'Jacobs, A. (2010). Creación de valor para el sector público, privado y social a través de la promoción de mercados de saneamiento en la base de la pirámide en el Perú. Tesis no publicada para optar al título de Magister en Administración, Universidad del Pacífico, Lima, Perú.

Cantillón, R. (1755). Essai sur la nature du commerce en general. Londres: Macmillan.

Celli, J.B. \& González, R.A. (2010). Marketbased initiatives for low-income sector and economic value creation. Socially Inclusive Business. Cambridge, MA: Harvard University Press.

Coase, R. (1937). The nature of the firm. Economica, 4(4), 386-405.

Drucker, P. (1985). Innovation and Entrepreneurship. New York, NY: Harper Collins.

Hébert, R.F \& Link, A.N. (1989). In search of the meaning of entrepreneurship. Small Business Economics, 1(1), 39-49.

Hébert, R.F. \& Link, A.N. (2006). The Entrepreneur as Innovator. The Journal of Technology Transfer, 31(5), 589-597.

Instituto Nacional de Estadística e Informática del Perú -INEI. (2007). Censos Nacionales 2007: XI de Población y VI de Vivienda. Disponibles en http://www. inei.gob.pe/biblioineipub/bancopub/Est/ Libo838/libro10/index.htm

Mair, J. \& Martí, I. (2004). Social entrepreneurship research: A source of explanation, prediction and delight (Working Paper 546, pp. 1-21). España: IESE.

Ogliastri, E. (2003). Alianzas cívicas y la empresa social: una introducción. Revista Latinoamericana de Administración, 31, 5-13.

Schumpeter, J.A. (1934). The theory of economics development. Cambridge, UK: Harvard University Press. 
Sen, A.K. (1984). The living standard. Oxford Economic Papers, New Series, 36(Supplement: Economic Theory and Hicksian Themes), 74-90.

Sirgy, M.K, Michalos, A.C., Ferriss, A.L., Easterling, R.A., Patrick, D. \& Pavot, W. (2006). The quality-of-life research movement: past, present, and future. Social Indicators Research, 76(3), 343-466.

Stevenson, H. (2006). A perspective on entrepreneurship. Harvard Business School Background Note, 384-131 (version revisada de version impresa en 1983).

Stevenson, H. \& Gumpert, D. (1985). The heart of entrepreneurship. Harvard Business Review, March-April, 85-94.

Thakor, A. \& Quinn, R. (2006). Competing Values Leadership: Creating Value in Organizations. Northampton, MA: Edward Elgar.

Van Praag, M. \& Versloot, P. (2007). What is the value of entrepreneurship? A review of recent research. Small Business Economics, 29(4), 351-382.

Weerawardena, J. \& Mort, G. (2006). Investigating social entrepreneurship: A multidimensional model. Journal of World Business, 41(1), 21-35.

Wei-Skillern, J., Austin, J.E., Leonard, H. \& Stevenson, H. (2007). Entrepreneurship in the social sector. Thousand Oaks, CA: Sage. World Bank. (2010). Iniciativa Creando Mercados de Saneamiento. Documentos de sistematización. Lima: Autores.

World Health Organization -wHo. (1986, noviembre). Ottawa Charter for Health Promotion. Documento no publicado presentado en First International Conference on Health Promotion, Ottawa, Canada.

World Health Organization -wHo. (2005). The Bangkok Charter for Health Promotion in a Globalized World. Documento no publicado presentado en Sixth Global
Conference on Health Promotion, Bangkok, Thailand. 


\section{Anexo 1. Factores que estimulan la inversión en saneamiento en la base de la pirámide}

\begin{tabular}{|c|c|}
\hline Factor & Contexto \\
\hline $\begin{array}{l}\text { Derecho a la dignidad } \\
\text { humana }\end{array}$ & $\begin{array}{l}\text { La valoración a la dignidad humana, reconocida en este caso en un respeto irrestricto } \\
\text { a la intimidad de actos naturales; pero en espacios reservados, limpios, seguros, con } \\
\text { comodidades básicas, que no generen contaminación ni se conviertan en focos de in- } \\
\text { fección, se convierte en una necesidad de las familias y en un indicador de bienestar } \\
\text { y calidad de vida, de preferencia valorado por las mujeres y los niños. El poseer un } \\
\text { servicio de saneamiento privado, cómodo y amplio, dentro o próximo al hogar y con } \\
\text { servicio de agua, que permita la realización de la limpieza de la persona -en toda su real } \\
\text { dimensión- genera igualmente en las familias la satisfacción de estar cuidando a sus } \\
\text { hijos, y una sensación de mejora de la calidad de vida que se refleja en la percepción de } \\
\text { un diferente status con respecto a aquellas familias que no lo poseen. }\end{array}$ \\
\hline
\end{tabular}

Socialización del concepto "saneamiento"

Servicios públicos básicos

Regularidad en los ingresos

Seriedad y labor de los promotores

Oferta integral de productos y servicios estructurados

Participación comunal como germen de la asociatividad
La acción de las políticas públicas con respecto al tema de salubridad y saneamiento tienen impacto en la población, no solo por la implementación concreta en campañas de promovidas por el gobierno central, regional o local, sino también por su componente comunicacional al divulgar de manera masiva conceptos claros y precisos de lo que significa el vivir una vida en condiciones de salubridad, eliminar centros de contaminación y llevar prácticas individuales y colectivas de higiene personal.

El hecho de contar con una red de alcantarillado público acelera la decisión de invertir en saneamiento. Una situación inversa se produce cuando por localización geográfica u otras razones no se cuenta con acceso a los servicios públicos básicos, dando prioridad a otras necesidades. Esto se posterga la instalación de baños hasta contar con red de alcantarillado público en sus zonas, o en su defecto, la labor de comunicación y promoción deben ofrecer otras alternativas de solución, como alternativas secas y pozos sépticos.

La frecuencia de ingresos no necesariamente se refiere a un empleo estable, también hace referencia a actividades económicas que generen ingresos como el autoempleo o negocios propios. Es posible, sin embargo, gracias a subvenciones del Estado, como los fondos de apoyo social para reducir la pobreza.

La labor de los promotores ayuda, guía y orienta a las familias en la toma de decisiones en la inversión para la instalación de baños en la vivienda. No solo fungen como meros vendedores, sino que representan a organismos públicos, privados o sin fines de lucro, donde la seriedad de las instituciones ofrece un elemento de garantía a la inversión que las familias desean ejecutar.

Se necesita una oferta de productos y servicios de saneamiento para el hogar, de tal manera que la decisión de inversión se plasme inmediatamente en el bien que será instalado en la vivienda, lo cual implica alianzas comerciales a fin de poseer en los locales de comercialización los stocks de materiales y kits de saneamiento necesarios. La estructuración del mercado de oferta es tan necesaria como la conformación incipiente del mercado de demanda.

Muchas de las actividades son desarrolladas bajo esquemas de participación o colaboración comunal, gracias a la socialización de la comunicación y al interés comunitario que se genera al hacer del tema de salud un aspecto importante de la vida comunal. La participación comunal para beneficio de todos es un elemento que influye en la decisión de inversión en saneamiento de las familias, porque se dan cuenta que el bienestar puede ser mayor que él solo individual.

Fuente: Elaboración propia a partir de los reportes de sistematización de la iniciativa CMs (World Bank, 2010). 
Anexo 2. Logros de aprendizaje. Creando el mercado de saneamiento: resultados del plan piloto en Cajamarca, Huaraz, Cuzco y Callaro

\begin{tabular}{|c|c|}
\hline $\begin{array}{l}\text { Componentes } \\
\text { del modelo }\end{array}$ & Logros de aprendizaje \\
\hline $\begin{array}{l}\text { Conformación de } \\
\text { alianzas estratégicas }\end{array}$ & $\begin{array}{l}\text { 1. Bajo el enfoque de mercado es conveniente destinar más esfuerzos en la participación } \\
\text { y liderazgo del sector privado que en el sector público. }\end{array}$ \\
\hline $\begin{array}{l}\text { Conformación de } \\
\text { comités locales de } \\
\text { gestión }\end{array}$ & $\begin{array}{l}\text { 2. Es mejor promover espacios de diálogo y participación de los actores para facilitar } \\
\text { información e interacción, que para facilitar las alianzas y desarrollar del mercado. }\end{array}$ \\
\hline $\begin{array}{l}\text { Empoderamiento de } \\
\text { actores en la CMS }\end{array}$ & $\begin{array}{l}\text { 3. Es clave identificar un sector público estratégico para impulsar el proceso y fortalecer } \\
\text { sus capacidades de gestión, sensibilizando y difundiendo desde el Gobierno Regional y } \\
\text { en paralelo hacia las autoridades e instituciones locales. }\end{array}$ \\
\hline \multirow[t]{2}{*}{$\begin{array}{l}\text { Planes de trabajo con- } \\
\text { junto }\end{array}$} & $\begin{array}{l}\text { 4. Las alianzas funcionan cuando sus integrantes participan con capacidad de decisión } \\
\text { y aporte directo de recursos (casos de Direcciones Regionales, Universidades). Las re- } \\
\text { uniones periódicas que involucran a los actores claves del Gobierno Regional, municipio, } \\
\text { empresas y ong, a través de un Comité Técnico o similar, aportan positivamente a la } \\
\text { marcha del Proyecto. }\end{array}$ \\
\hline & $\begin{array}{l}\text { 5. El Gobierno Municipal, en cumplimiento de las funciones que señala la ley sobre sa- } \\
\text { neamiento, salubridad y salud, además del desarrollo económico local, debe fortalecer } \\
\text { aún más las capacidades de los microempresarios que producen sanitarios de granito, } \\
\text { mejorando su tecnología y calidad para una mayor participación en el mercado. }\end{array}$ \\
\hline $\begin{array}{l}\text { Impulso a la demanda } \\
\text { Movilización comu- } \\
\text { nitaria }\end{array}$ & $\begin{array}{l}\text { 1. Sensibilizar la propuesta desde espacios de concertación ya establecidos y legitimados } \\
\text { por los líderes y miembros una localidad contribuye a favor de un mayor involucramien- } \\
\text { to de los actores participantes, impacto y sostenibilidad del modelo. En estos espacios se } \\
\text { concreta la alianza público - privado - social. }\end{array}$ \\
\hline Movilización educativa & $\begin{array}{l}\text { 2. El diseño de un proyecto con los actores que participarán en su ejecución, tanto en el } \\
\text { nivel nacional, regional y local, aportará en la comprensión del modelo, mayores compro- } \\
\text { misos y mejores resultados e impactos en la población objetivo }\end{array}$ \\
\hline \multirow{4}{*}{$\begin{array}{l}\text { Promoción de pro- } \\
\text { ductos y servicios de } \\
\text { saneamiento }\end{array}$} & $\begin{array}{l}\text { 3. El desarrollo de diversas formas y metodologías de información, comunicación y de } \\
\text { amplitud multisectorial para llegar a las familias es la forma más adecuada y lograr mejor } \\
\text { sensibilización. }\end{array}$ \\
\hline & $\begin{array}{l}\text { 4. Los incentivos efectivos por parte del gobierno local (aportes en materiales) o del sec- } \\
\text { tor privado (descuentos por compra por grupos, compra por partes) facilitan la decisión } \\
\text { de compra. }\end{array}$ \\
\hline & $\begin{array}{l}\text { 6. La promoción que mejores resultados ha logrado es aquella que hace participar a la } \\
\text { población y que viene del sector privado (ferias, visitas domiciliarias). }\end{array}$ \\
\hline & $\begin{array}{l}\text { 7. La autoconstrucción es un mecanismo de las familias para reducir sus costos pero no } \\
\text { consideran los riesgos de la calidad; es decir, se valora el precio y no la calidad. }\end{array}$ \\
\hline
\end{tabular}

(continúa) 
Anexo 2. Logros de aprendizaje. Creando el mercado de saneamiento: resultados del plan piloto en Cajamarca, Huaraz, Cuzco y Callaro (continuación)

\begin{tabular}{|c|c|}
\hline $\begin{array}{l}\text { Componentes } \\
\text { del modelo }\end{array}$ & Logros de aprendizaje \\
\hline Impulso a la oferta & $\begin{array}{l}\text { 1. El desarrollo de nuevos productos no es automático, es intensivo en capital y profesio- } \\
\text { nales especializados que en una primera etapa el sector privado debe ser asistido para su }\end{array}$ \\
\hline \multirow{2}{*}{$\begin{array}{l}\text { Desarrollo de opciones } \\
\text { técnicas de saneamien- } \\
\text { to básico }\end{array}$} & posterior adaptación y masificación. \\
\hline & $\begin{array}{l}\text { 2. El producto de saneamiento que mayor demanda ha tenido es el que emplea los mate- } \\
\text { riales y la mano de obra de las zonas y la que se construye por etapas (Ej. Cajamarca, Baño }\end{array}$ \\
\hline Planes de negocio con & Lluvia, de adobe más de $80 \%)$. \\
\hline (ferreterías) & $\begin{array}{l}\text { 3. Es más conveniente económica y técnicamente promover y capacitar a nuevos prov- } \\
\text { eedores de servicios especializados en saneamiento (maestros de obra) que puedan ser }\end{array}$ \\
\hline $\begin{array}{l}\text { Diseño e implement- } \\
\text { ación de módulos de } \\
\text { capacitación en servi- }\end{array}$ & $\begin{array}{l}\text { de las propias localidades o las más cercanas. Los jóvenes son más proactivos y compro- } \\
\text { metidos con la iniciativa. }\end{array}$ \\
\hline cios de saneamiento & 4. Es más efectivo contar con promotores que a su vez sean maestros de obra. \\
\hline $\begin{array}{l}\text { Formación de op- } \\
\text { eradores comunitarios } \\
\text { (gasfiteros) }\end{array}$ & $\begin{array}{l}\text { 5. La venta del producto como un todo (materiales más mano de obra) tiene menor de- } \\
\text { manda que la venta del productos por etapas. }\end{array}$ \\
\hline Acceso al crédito & $\begin{array}{l}\text { 1. El desarrollo de productos de crédito está condicionado al incremento de la demanda } \\
\text { y tamaño del mercado, por tanto no es conveniente insistir desde el inicio con el sector }\end{array}$ \\
\hline Involucramiento del & financiero para que baje sus tarifas o readecue línea al nuevo producto. \\
\hline $\begin{array}{l}\text { Promoción de nuevos } \\
\text { instrumentos finan- } \\
\text { cieros accesibles a los }\end{array}$ & $\begin{array}{l}\text { 2. La promoción de diferentes alternativas de financiamiento, e incluso una forma de } \\
\text { banca comunal, favorece la competencia y facilita la oferta de mejores condiciones fi- } \\
\text { nancieras. }\end{array}$ \\
\hline pobres & $\begin{array}{l}\text { 3. El crédito ha tenido más aceptación en el sector periurbano que en el rural por temas } \\
\text { de dinámica económica, experiencia crediticia y de articulación al mercado. }\end{array}$ \\
\hline $\begin{array}{l}\text { Promoción de otras } \\
\text { formas de financia- } \\
\text { miento no formal: } \\
\text { autofinanciamiento } \\
\text { comunitario }\end{array}$ & $\begin{array}{l}\text { 4. La segmentación del mercado y la información de los perfiles del cliente (capacidad de } \\
\text { pago, propiedad, activos animales, micro negocios) orienta una mejor intervención de } \\
\text { las entidades financieras. }\end{array}$ \\
\hline
\end{tabular}

Fuente: Elaboración propia a partir de los reportes de sistematización de la Iniciativa CMs (World Bank, 2010). 


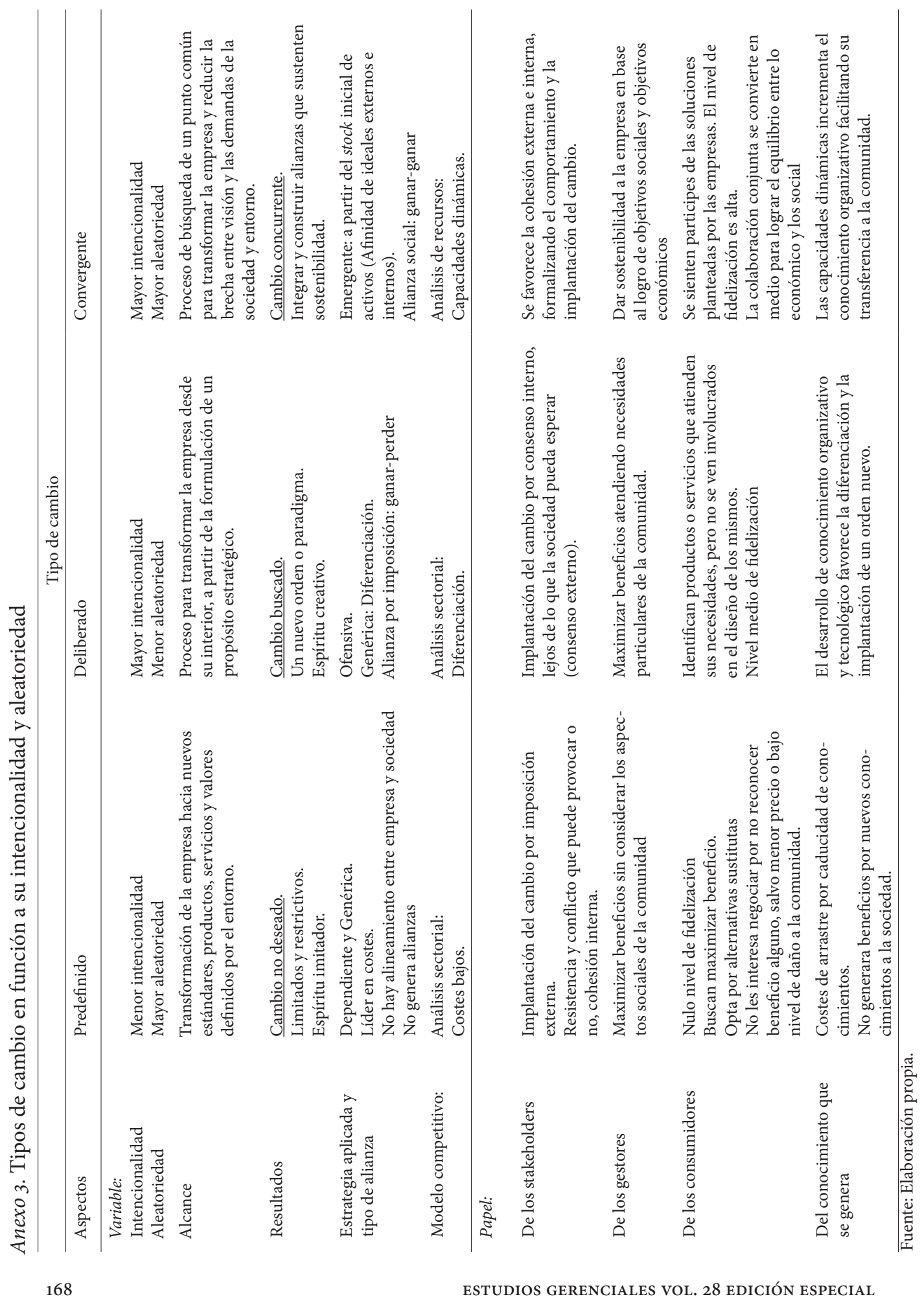

